\title{
Trois décennies de partenariat avec des personnes fragiles
}

\section{Jean Martin}

Dr méd., membre de la rédaction

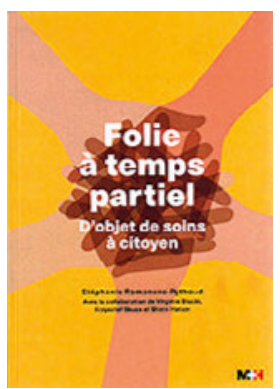

Stéphanie Romanens-Pythoud, avec la collaboration de V. Stucki, K. Skuza et Sh. Hatam

Folie à temps partiel - D'objet de soins à citoyen

Chêne-Bourg: Médecine et Hygiène; 2017.

208 pages. $22 \mathrm{CHF}$.

Cette somme substantielle décrit l'histoire riche et parfois mouvementée, en Suisse romande, des mouvements d'accompagnement et soutien aux patients psychiques, dans un sens d'empowerment. Le livre est publié à l'occasion des 30 ans du Graap (Groupe d'accueil et d'action psychiatrique) vaudois, conjointement avec ses homologues fribourgeois (afaap) et neuchâtelois (anaap).

«Les associations dérangent... et c'est indispensable! Il leur appartient de mettre le doigt sur les failles des systèmes médicaux et sociaux, de dénoncer les pratiques inacceptables. Mais elles devraient aussi rêver l'avenir», dit la juriste Béatrice Despland dans sa préface. Jeune médecin cantonal lors de la création du Graap, je peux confirmer qu'il a parfois dérangé... Nous avons eu des échanges vifs, notamment à propos de contention en psychiatrie. Mais, avec l'autorité sanitaire et avec les cadres psychiatriques, nous avons reconnu le caractère constructif et la volonté de partenariat du Graap. Au chapitre 1 est rappelée la mouvance anti-psychiatrique des années 1970, y compris la contestation vive, surtout à Genève, des électrochocs - qui a mené à la cessation de la pratique dans ce canton.

Madeleine Pont, cofondatrice du Graap, a été une force majeure dans ces développements; travaillant dans les années 1970 pour Pro Mente Sana, elle «a le sentiment que les assistants sociaux ne touchent pas leur cible par le seul travail individuel avec les clients. Elle est convaincue qu'il faut responsabiliser les personnes concernées et qu'en groupe elles pourront résoudre elles-mêmes leurs difficultés.» A noter que, après vingt ans d'activité, le Graap a connu une période difficile, liée à un fonctionnement très (trop?) démocratique compliquant la gestion d'une association qui avait beaucoup grandi. Les problèmes ont pu être surmontés par la création d'une fondation aux côtés de l'association. "Cela ne s'est pas fait sans drames, départs et licenciements mais cette transformation était indispensable.»

Une section est consacrée à la place et aux rôles des proches des patients, sous le titre «Du proche coupable au proche partenaire». Il est vrai qu'on voit/voyait à leur égard une certaine méfiance. Témoignage: «Il y avait énormément de culpabilisation des familles dans les années 1980. Quand notre fille avait moins de 18 ans, oui, on était informé. Mais dès qu'elle a été majeure, ça a été fini.» Questions qui restent actuelles entre autonomie du patient, secret médical et intérêts compréhensibles des proches. Les associations d'action pour la santé psychique ont élaboré une Charte des proches, fruit des réflexions menées au cours d'ateliers citoyens et publiée en 2013, qui a représenté un pas important.

Dans les conclusions, à noter la contribution de Shirin Hatam, juriste à Pro Mente Sana, sur le nouveau droit de la protection de l'adulte entré en vigueur en 2013. Elle donne des précisions importantes sur les apports de ce nouveau droit, ne ménageant pas certaines critiques, y compris sur un caractère pas assez nuancé, voire régressif - et interprété de manières diverses selon les cantons. Est discutée la question du travail et l'importance qu'on lui accorde: «Peut-on pleinement exister, être épanoui, sans travail salarié?» La réponse des auteurs est clairement oui. Beaucoup de ce que les personnes font jour après jour (y compris le travail sur soi et sa maladie) est un vrai travail.

Dans les dernières pages, un point majeur: «Dans une société partagée entre les principes de la liberté individuelle et ceux du risque zéro, nos organisations doivent être en faveur d'une politique du risque mesuré en offrant de larges places à la créativité et à la co-construction. La focalisation actuelle sur le risque zéro se répercute gravement [négativement] sur les justiciables.»

En résumé: une présentation large et diversifiée sur des démarches pionnières et militantes de grande valeur, au plan médico-social et sociétal, depuis quelques décennies en Suisse romande. 